\title{
The Protein Kinase A (PKA) Intracellular Pathway and Androgen Receptor: A Novel Mechanism Underlying the Castration-resistant and Metastatic Prostate Cancer
}

\author{
Martuza Sarwar and Jenny L Persson* \\ Experimental Cancer Research, Department of Laboratory Medicine, Lund University, Sweden
}

\begin{abstract}
Androgen deprivation therapy (ADT) in the form of medical or surgical castration has been used as the standard therapy for localized and advanced prostate cancer (PCa). Although ADT leads to the initial regression of tumors, the recurrence of more aggressive PCa, also termed as castration-resistant PCa (CRPC) is inevitable. Androgen receptor (AR) and its associated network have been proposed to play central role in the progression of CRPC. Given that CRPC exhibits the nature of heterogeneity and complexity, multiple cellular pathways may cooperatively promote progression of CRPC and render the tumors insensitive to therapy. In this article, we will review some of the recent findings and clinical interventions to identify the novel targets and alternative signaling pathways associated with AR that may allow the aggressive forms of PCa to recur and become resistance to therapy. We will discuss about the role of the cyclic adenosine monophosphate (cAMP) activated protein kinase A (PKA) pathway in the progression of CRPC. The emerging evidence suggests that several key factors in PKA signalings may play important roles in the recurrence and treatment response of CRPC, and that PKA pathways may serve as potential diagnostic and predictive biomarkers for CRPC. We will also update with the information on the novel therapeutic strategies that have been designed and tested in laboratories to inhibit PCa growth by targeting both AR and PKA pathways. Understanding of the molecular mechanisms underlying the progression of CRPC and treatment resistance will provide novel insight for effective treatment of CRPC.
\end{abstract}

Keywords/Abbreviations: Prostate cancer (PCa); Androgen deprivation therapy (ADT); Castration resistant prostate cancer (CRPC); Cyclic AMP or 3'-5'-cyclic adenosine monophosphate (cAMP); Protein kinase A (PKA); Androgen receptor (AR); Phosphodiesterase enzymes (PDE)

\section{Introduction}

Prostate cancer (PCa) is one of the most common forms of cancer in men in the western countries [1]. As the growth of primary prostate cancer cells rely on circulating androgens, androgen deprivation therapy (ADT) in the form of medical or surgical castration has been used as the standard therapy for the treatment of localized and advanced PCa [2]. Although ADT leads to an initial regression of the tumor, in most of the cases PCa recurs as an aggressive form and becomes independent of androgen and this type of $\mathrm{PCa}$ is termed as castrationresistant prostate cancer (CRPC) [3]. The metastatic CRPC is lethal and the overall survival is low [4]. Because CRPC exhibits heterogeneity and complexity, which may lead to the treatment resistance for some CRPC patients, it is therefore an urgent need to define multiple cellular pathways that may cooperatively promote progression of CRPC and render the tumors insensitive to therapy. Further, it is also important to develop novel therapeutic strategies for the effective treatment of CRPC. In this review we will discuss some of the recent findings and clinical interventions with specific focus on PKA pathways and its functional interplay with AR to promote recurrence and treatment resistance of PCa.

\section{AR signaling pathways in the development of CRPC}

Despite extensive research in the past decades, the molecular mechanism underlying the progression of CRPC is still poorly understood. It is widely accepted that androgen receptor (AR) plays a central role in the development of CRPC, despite the depleted or low levels of circulating androgen, CRPC still expresses AR and AR responsive prostate specific antigen (PSA) [5]. Further, most of the CRPCs displayed elevated level of AR [6], thus AR is likely to be responsible for sensitizing PCa to low level of androgen and promote recurrence of CRPC. In addition, in the absence of androgen, alternative growth stimulations may replace the effect of androgen to promote tumor growth. It has been shown that growth factors such as epidermal growth factor (EGF), interleukin-6 (IL-6) and the neuropeptide including bombesin and gastrin releasing peptides can activate AR in the absence of androgen in prostate cancer cell lines [7-9]. Moreover, accumulating evidence also suggests that multiple cellular mechanisms such as deregulation of the phosphatidylinositol 3-kinase (PI3-K)-Akt pathway, overexpression of the anti-apoptotic B-cell lymphoma 2 (Bcl2) gene as well as neuroendocrine differentiation may contribute to the progression to CRPC [10-12]. Because AR is a ligand dependent nuclear transcription factor that mediates the action of testosterone and dihydrotestosterone (DHT), the altered intracellular localization may affect the compositions of $\mathrm{AR}$ associated networks and render

\footnotetext{
${ }^{*}$ Corresponding author: Jenny L Persson, Division of Experimental Cancer Research, Department of Laboratory Medicine, Lund University, Clinical Research Center, 205 02, Malmö, Sweden, Tel: 46-40-391106; Fax: 46-40-391222; E-mail: jenny_l.persson@med.lu.se
}

Received November 07, 2011; Accepted December 04, 2011; Published December 06, 2011

Citation: Sarwar M, Persson JL (2011) The Protein Kinase A (PKA) Intracellular Pathway and Androgen Receptor: A Novel Mechanism Underlying the Castration-resistant and Metastatic Prostate Cancer. J Cancer Sci Ther S5:003. doi:10.4172/1948-5956.S5-003

Copyright: @ 2011 Sarwar M, et al. This is an open-access article distributed under the terms of the Creative Commons Attribution License, which permits unrestricted use, distribution, and reproduction in any medium, provided the original author and source are credited. 
the cells hypersensitive to low androgen. It has been shown that AR remains inactive in the cytoplasm when bound to the heat shock proteins (HSP) such as HSP90, HSP70 and Hsp56. Binding of the hormone (testosterone and DHT) leads to conformational changes of the AR, releasing AR from the HSP-AR complexes and affects the subcellular translocation of AR [9]. Thus, the alterations or disruption of the multiple pathways that are associated with AR nuclear transport may also contribute to the progression of CRPC.

\section{The molecular composition of the PKA signaling pathways}

Cyclic adenosine monophosphate (cAMP) activated protein kinase A (PKA) pathway has been implicated in the progression to CRPC [13]. It has been shown that PKA pathway was involved in the progression of PCa through activation of protein kinase $\mathrm{B}(\mathrm{PKB})$ and through expression of $\mathrm{Bcl}-2$ as well as via induction of neuroendocrine differentiation. PKA is a serine/threonine kinase that reversibly phosphorylates numerous proteins in the cytoplasm and in the nuclear compartment and it is dependent on cAMP for its activity [14]. The cAMP is the classical second messenger discovered in 1958 and has been shown to play a role in metabolism, cellular growth, differentiation, gene expression and apoptosis [14]. Intracellular cAMP is produced by the enzyme adenylyl cyclase (AC) and is dependent on hormonal stimulation via the stimulatory G protein coupled receptor [14]. The PKA exists as a tetrameric holoenzyme consisting two regulatory $(\mathrm{R})$ and two catalytic (C) subunits forming a holoenzyme $\mathrm{R}_{2} \mathrm{C}_{2}$ [15]. As a result of either homo- or heterodimerization of different $\mathrm{R}$ subunits, a diversity of PKA isozymes including PKAI $\left(\mathrm{R} 1 \alpha_{2} \mathrm{C}_{2}, \mathrm{R} 1 \beta_{2} \mathrm{C}_{2}\right)$ and PKAII $\left(\mathrm{R} 2 \alpha_{2} \mathrm{C}_{2}\right.$, $\mathrm{R} 2 \beta_{2} \mathrm{C}_{2}$ ) are formed $[14,15]$. The $\mathrm{R}$ subunits are the major intracellular receptors of cAMP. Binding of the CAMP by the R subunits leads to the release of $\mathrm{C}$ subunits from the $\mathrm{R}-\mathrm{C}$ complexes, and allow the $\mathrm{C}$ subunits to phoshorylate downstream substrates [15]. The $C$ subunits $C \alpha, C \beta$, and $\mathrm{C} \gamma$ are coded by the genes PRKACA, PRKACB and PRKACG respectively $[14,15]$. PKA pathways control the intracellular calcium, cell proliferation, inflammation and transcription [16]. PKA exerts the effects of cAMP on several key transcriptional factors, among them the cAMP response element (CRE)-binding protein 1 (CREB/CREB1) is the principle mediator. Phosphorylation of CREB by PKA at serine 133 is required for the activation of CREB1 [17]. Phosphorylated CREB (pCREB) can modulate the expression of a large number of genes that are involved in cell growth and survival $[18,19]$. The family of cyclic nucleotide phosphodiesterase isozymes (PDEs) regulates intracellular levels of cAMP and cGMP. There is a reciprocal regulation between PDEs and PKA pathways. The cAMP specific PDEs including PDE4, PDE7 and PDE8 family of proteins play important roles in controlling the activity of the PKA pathway [20]. PKA in turn mediates the activities of the PDEs. PDE4 family of proteins is mainly expressed in inflammatory cells, brain, endothelial cells and cardiovascular tissues. PDE4 activity is mediated by PKA through phosphorylation. Upon the hormonal stimulation, the increased level of cAMP will lead to the increased activity of PDE4 [21]. This suggests that PKA phosphorylation may bridge the link between cAMP and PDE4. Multiple isoforms of the PDE4 family namely the PDE4A, PDE4B, PDE4C and PDE4D have been shown to play important role in cell growth and survival. Recruitment of PDE4 during T cell activation is an important event to enhance the T-cell immune response [22]. PDE4, 7 and 8 are critical components of cAMP-dependent PKA signaling. However, the expression patterns and role of PDEs in pathogenesis PCa remains to be investigated. Another family of PKA associated proteins, termed as the A-Kinase anchoring proteins (AKAPs) can bind to the regulatory subunits of PKA and this interaction is thought to be responsible for the subcellular localization of PKA. The AKAPs also aids to the termination of PKA signaling by bringing PDE isozymes to degrade cAMP $[23,24]$.

The cAMP has long been described as a key regulator in cell growth and differentiation. Its role in controlling tumor growth was demonstrated in a series of studies where the cAMP analogos were able to inhibit tumor growth and induce differentiation in various types of tumors $[25,26]$. Site selective cAMP analog designated as 8-CIcAMP has potent inhibitory effect on breast cancer and colon cancer cell lines [27]. Because 8-CI-cAMP facilitates its effect on cell growth and differentiation via PKA type I and type II isozymes (PKAI and PKAII) that are the result of the interactions between $\mathrm{R}$ subunits and $\mathrm{C}$ subunits, the potential role of PKA pathways in tumorigenesis has been considered [28]. Due to that cAMP acts as positive and negative intracellular regulators depending on the physiological conditions, hormonal status and cell types [28], the cAMP-dependent PKAI and PKAII also have dual function of either inhibiting or promoting cell growth and differentiation. Not until a decade ago, the distinct function of PKAI and PKAII in controlling cell proliferation was defined.

In normal cells, while PKAI is transiently expressed at high levels in response to physiological or hormonal stimuli, PKA-II is preferentially expressed in differentiated tissues. In cancer cells, PKAI is constitutively overexpressed and is associated with poor prognosis in various types of human cancers $[29,30]$. This suggests that there is a balance between the expression levels and activities of PKAI and PKAII in normal tissues, and this balance is disrupted during tumorigenesis. Therefore 8-CI-cAMP may restore the balance of cell growth from malignancies by suppression of PKAI and induction of PKAII. Expression of PKAI and PKAII has been observed in normal prostate tissues. The activity and expression level of PKAI is dependent on the level of hormones, as PKAI activity decreases by $50 \%$ in ventral prostate three days postcastration, whereas PKAII level shows little changes [28,31]. This suggests that there is a functional link between the PKAI protein levels and hormone production.

\section{PKA pathways in the development of CRPC}

The expression of several PKA subunits have been examined in several prostate cancer cell lines [25,32] and in PCa specimens [30] from several patient cohorts. Overexpression of PKARIa subunit resulted in the induction and up-regulation of global expression of genes that are required for proliferation and tumor progression in the aggressive PC3M cells [32]. Conversely, proto-oncogens and genes for tyrosine and serine/threonine kinases that are overexpressed in tumors were specifically downregulated following the treatment with antisense to PKARIa [33,34]. PKARIa subunit overexpression showed prognostic values to predict outcome in prostate cancer patients treated with radiotherapy (RT) with or without short-term androgen deprivation therapy. In a patient cohort consisting of 313 cases in the RTOG 9202 study, overexpression of PKARIa subunit was shown to correlate with increased risk of failure after combination treatment with ADT and RT. Overexpression of PKARIa was also associated with distance metastasis. This suggests that the length of $\mathrm{AD}$ to patient outcome is affected by PKARIa overexpression [30,35]. The more recent studies have demonstrated that combined knockdown of AR and inhibition of PKARIa enhanced growth arrest of prostate cancer cells [36]. Further, 
inhibition of PKARIa via antisense molecules also inhibited growth of PCas in cell lines and in xenograft mice, suggesting an important role for PKARIa in promoting the growth of PCa $[37,38]$.

The role of PDEs in the progression of PCa was studied in PCa cell lines including PC3, DU145 and LNCaP cells. Treatment of LNCaP cells with the non-selective phosphodiesterase inhibitor, papaverine inhibited proliferation and invasive potential and induced terminal differentiation in LNCaP cells, which is correlated with an intracellular cyclic AMP-mediated pathway [39]. Expression of cAMP specific phosphodiesterases such as PDE4B and PDE4D are functionally linked to PKA and AR as well. Overexpression of PDE4D was shown to result in a significant reduction of androgen mediated activation of $A R$ in LNCaP cells [40]. And more recently, PDE4B was found to be downregulated in advanced prostate cancer and targeted knockdown of PDE4B promoted androgen independent growth in LNCaP cells [41].

Expression of genes encoding PKA-related A kinase anchoring proteins AKAP3 and AKAP4 was observed in PCa tumor tissues but was not detected in normal prostate tissues [42]. Recently AKAP4 was shown to be higly expressed at the protein level in tumors from PCa patients [43]. Overexpression of CREB1, the transcriptional factor associated with PKA pathways are the common events in various types of cancer including $\mathrm{PCa}$, breast cancer, non-smallcell lung cancer and acute leukemia [44]. The transcription factor CREB has also been implicated in PCa metastasis, as bone metastatic tissues displayed positive positive staining for phosphorylated CREB (pCREB), while their normal counterpart had no detectable pCREB activity [45]. Further evidence also suggested that CREB may promote bone metastasis of PCa by modulating the expression of multiple genes required for angiogenesis including VEGF and HIF-1 [45]. The elevated level of PKIB (cAMP-dependent protein kinase inhibitor-beta), was observed specifically in CRPCs and in aggressive PCas. There was a direct interaction between PKIB and PKA subunit $C$, as knockdown of PKIB in PCa cells altered the subcellular localization of PKA C subunit. PKIB enhanced phosphorylation of Akt at Ser473 by mediating the activity of PKA-C kinase. These findings suggest that PKIB and PKA subunit $\mathrm{C}$ may play an important role in the progression of PCa by mediate PI3/Akt pathways [46].

\section{Modulation of AR action via PKA}

It has been shown that PKA controls AR function partly through its ability to modulate the subcellular localization of AR [47]. Inhibition of PKA by H89 (selective inhibitor of PKA) led to the cytoplasmic sequestration AR in the presence of R1881 (a synthetic androgen) in LNCaP cells. It is suggested that PKA may modulate nuclear entry of AR by phosphorylating the HSP90. The HSP90 is responsible for retaining the AR in the cytoplasm, PKA mediated phosphorylation of HSP90 relieves AR from its grip and aids in nuclear translocation [48]. However, the role of nuclear entry of AR that is controlled by PKA pathways in the progression of CRPC remained to be investigated.

In androgen-independent PC-3 cells, induction of AR expression in combination with stimulation of PKA pathways by forskolin (an enhancer of cAMP) led to the activation of AR in an androgenindependent fashion [13,49]. Moreover, Sadar et al. [50] have shown that forskolin stimulation led to the induction of androgen responsive PSA gene and other ARE driven reporter constructs in LNCaP cells as well as in PC-3 cells when transfected with functional AR. This finding suggests that activation of AR target genes is in part mediated by PKA dependent-mechanisms. Further PKA pathway is likely to be responsible for the phosphorylation of $\mathrm{AR}$ and regulate the level of $\mathrm{AR}$ in PCa cells. Kim et al. [51] have demonstrated that forskolin enhanced PSA expression in the presence of DHT via CREB in LNCaP cells. The CREB was activated by PKA mediated phosphorylation at serine 133, which then binds to the CRE present at the $5^{\prime}$ regulatory region of PSA gene. The activated CREB may serve as co-activator of AR on the transcriptional activity of PSA [52]. Taken together, in the absence of androgen or at the condition of low androgen levels, PKA is able to regulate AR protein level and activate AR target genes through multiple mechanisms.

\section{Crosstalk between PKA and AKT pathway in PCas}

The PI3K-Akt pathway plays an important role in malignant or aggressive phenotype of hormone independent prostate cancer, as elevated phosphorylated AKT is correlated with high Gleason grade [53]. It is well established that AKT plays an important role in proliferation, survival and cancer metastasis. The AKT is also termed as protein Kinase $B(\mathrm{PKB})$ due to its resemblance to PKA. AKT is a family of serine/threonine kinases comprising AKT1, AKT2, and AKT3 [54]. For activation of AKT, phosphorylation at threonine 308 (Thr 308) and at serine 473 (Ser 473) is required. Phosphoinositide dependent kinase 1 (PDK1) is known to phosphorylate Thr 308. However, up to date, kinases that are responsible to phosphorylate AKT at Ser 473 have not been fully characterized. There might be a possibility that PKA is the second kinase and studies from Chung et al. [46] suggested that $C$ subunit of PKA (PKA-C) may phosphorylate AKT at Ser 473.

Another component of the AKT pathway, the PI3K is also a substrate for PKA. The regulatory subunit of the PI3K, the p85a was shown to be phosphorylated by PKA at serine 83 (Ser-83) in the rat thyroid cell line FRTL- 5 as well as in mouse fibroblast NIH3T3 cells [55]. The isoforms of GSK-3 that are downstream of the PI3-K-Akt pathway are also the substrates of PKA [56]. Another level of crosstalk between PKA and AKT is mediated via CREB. Besides its activation by PKA, CREB can also be activated through phosphorylation at serine 133 by AKT. AKT mediated phosphorylation of CREB leads to the recruitment of the co-activator (CBP) and induces expression of antiapoptotic genes such as BCL-2 [53].

\section{PKA pathways and AR in neuroendocrine differentiated PCas and therapy resistance}

Neuroendocrine cells (NE) cells with the dual property of secretory and paracrine or autocrine function comprises only a minor portion of the normal prostate gland. The NEs are thought to play a role in androgen-independent growth of $\mathrm{PCa}[57,58]$. Increase in the number of NE cells was shown to correlate with tumor progression [59]. It was suggested that hormone therapy is ineffective in eliminating these NE cells, as they are likely androgen independent, thus NE tumor cells may contribute to the recurrence of CRPC and treatment resistance [60]. The enriched NE may establish paracrine or autocrine network to promote androgen independent growth. Furthermore, the NE cells secretes neuropeptides such as calcitonin, gastrin releasing peptide and vasoactive intestinal peptide (VIP) which can increase proliferation of PCa cells [50,61]. Accumulating evidence supports that the PCa cells can differentiate into the NE cells via the process termed as the neuroendocrine transdifferentiation (NED) under condition such 
as androgen deprivation [59]. Sustained activation of PKA has been correlated with NED of PCa cells. The overexpression of the Ca subunit of PKA as well as stimulation of forskolin led to the neuroendocrinelike differentiation, accompanied by the expression of neuron specific enolase (NSE) and morphological change such as neurite extension in LNCaP cells [62]. It has been shown that LNCaP cells constitutively expressing PKA catalytic subunit promoted tumorigenic ability and tumor growth in nude mice under androgen deprivation condition [63].

The potential utility of the CAMP/PKA pathways as biomarkers for predicting therapy or as drug targets for designing therapeutic interventions for treatment of CRPC

It has been shown that overexpression of the subunit of PKAR1a is related with poor outcome of prostate cancer patients [64]. Further, patients with elevated level of PKAR1a showed poor response to radiation therapy along with short term androgen withdrawal, indicating the diagnostic and prognostic significance of R1a [65]. Radiation therapy has been used to treat locally advanced PCa with the effect similar to ADT therapy [66]. Expression of PKAR1a has been shown to be clinically important to predict the failure of radiation therapy (RT) alone or with ADT therapy in combinations in several large clinical trials [67]. Recently, Hensley et al. [68] have shown that, antisense molecule targeting the RIa subunit of PKA in combination with ADT and radiation therapy significantly increased apoptosis in vitro as well as in vivo. Similarly, it has been shown that combined knockdown of AR and R1a subunit of PKA enhanced growth arrest of prostate cancer cell in comparison to AR knockdown alone [36]. Moreover, the effect of antisense targeting of R $1 \alpha$ subunit of PKA in combination with docetaxel has already been tested in a Phase I clinical trial with positive results $[37,38]$. Since higher concentration of intracellular cAMP level induces cell cycle arrest and attenuation of growth in various cell types [50], agents that increases cAMP level such as phosphodiesterase (PDE) inhibitors has been tested in prostate cancer cell lines. Nonselective PDE inhibitor such as papaverine led to growth arrest and differentiation of LNCaP cell line [39,50].

As noted above, tumor inhibitory effect of cAMP analog designated as 8-CI-cAMP has been demonstrated in various types of cancer cell lines including cancers of breast, lung, colon and leukemia $[26,28,69]$. Additionally PKAI-selective cAMP analogs (8-PIP-cAMP and 8-HACAMP) have been developed and tested on several human cancer cell lines including ARO, NPA and WRO cells. The cAMP analog 8-ClcAMP consistently inhibited cell growth, treatment with the PKAIselective cAMP analogs 8-PIP-cAMP and 8-HA-cAMP also induced growth arrest. Interestingly, PKAI-selective cAMP analogs induced growth arrest in cells carrying the BRAF oncogene, whereas 8-ClcAMP induce apoptosis, apparently through activation of the p38 MAPK pathway [70]. This finding suggests that 8-Cl-cAMP and the PKA I-selective cAMP analogs both have the growth inhibitory effects but act through different mechanisms. Given that cAMP analog 8-ClcAMP and PKAI-selective cAMP analogs have shown promising effects on various types of aggressive cancer cells, it will be interesting to test these compounds in PCa cell lines and in PCa xenograft models.

\section{Concluding Remarks and Future Directions}

As discussed above, we have presented the emerging novel evidence on that PKA may play important role in the progression of
CRPC and that PKA may regulate the AR activity and cross-talk with AR to enhance tumor cell growth and to render PCa cells insensitive to therapy. We also addressed the fact on that the molecular mechanisms underlying the functional interplay between the PKA pathways and AR is poorly understood. Only a few studies have been performed in using clinical samples from PCa patient cohorts. Expression patterns and levels of the key factors in PKA pathways in CRPC specimens and in metastatic lesions remained largely unknown. Because prostate cancer is a heterogeneous disease and multiple signal transductions cooperatively promote growth and survival of the cancer cells. It will be interesting to determine whether different PKA subunits and their related proteins such as AKAPs could be used as novel biomarkers for predicting treatment response and outcome of CRPC. The transcription factor CREB1 appeared to be a potential therapeutic target, as the knockdown of CREB in human leukemic cells was able to attenuate of proliferation and promote tumor cell death $[45,71]$, it will be important to examine these strategies in prostate cancer cells. Given that preclinical and laboratory studies have shown that targeting AR and PKA pathways in combination inhibited growth of PCa cells, it will be interesting to further develop and validate such novel therapeutic strategies for treatment of CRPC in large scales of studies. Understanding of the molecular mechanisms underlying the progression of CRPC and treatment resistance will provide novel insight for effective treatment of CRPC.

\section{Acknowledgements}

This work is supported by the grant from the Swedish Cancer Foundation, the Swedish National Research council, the Government Health Grant, the Lund University Medical Faculty Grant, the Malmö Cancer Foundation, the Skåne University Hospital Foundation to JLP.

\section{References}

1. Wegiel B, Evans S, Hellsten R, Otterbein LE, Bjartell A, et al. (2010) Molecular pathways in the progression of hormone-independent and metastatic prostate cancer. Curr Cancer Drug Targets 10: 392-401.

2. Shen MM, Abate-Shen C (2010) Molecular genetics of prostate cancer: new prospects for old challenges. Genes \& Dev 24: 1967-2000.

3. Dutt SS, Gao AC (2009) Molecular mechanisms of castration-resistant prostate cancer progression. Future Oncol 5: 1403-1413.

4. Attar RM, Takimoto CH, Gottardis MM (2009) Castration-Resistant Prostate Cancer: Locking up the Molecular Escape Routes. Clin Cancer Res 15: 3251 3255.

5. Lonergan PE, Tindall DJ (2011) Androgen receptor signaling in prostate cancer development and progression. J Carcinog 10: 20.

6. Gioeli D (2005) Signal transduction in prostate cancer progression. Clin Sci 108: $293-308$

7. Malinowska K, Neuwirt H, Cavarretta IT, Bektic J, Steiner H, et al. (2009) Interleukin-6 stimulation of growth of prostate cancer in vitro and in vivo through activation of the androgen receptor. Endocr Relat Cancer 16: 155-169.

8. Kung HJ, Evans CP (2009) Oncogenic activation of androgen receptor. Urol Oncol 27: 48-52

9. Grossmann ME, Huang H, Tindall DJ (2001) Androgen Receptor Signaling in Androgen-Refractory Prostate Cancer. J Natl Cancer Inst 93: 1687-1697.

10. Li L, Ittmann MM, Ayala G, Tsai MJ, Amato RJ, et al. (2005) The emerging role of the PI3-K-Akt pathway in prostate cancer progression. Prostate Cancer Prostatic Dis 8: 108-118.

11. Catz SD, Johnson JL (2003) BCL-2 in prostate cancer: A minireview. Apoptosis 8: 29-37.

12. Nelson EC, Cambio AJ, Yang JC, Ok JH, Lara PN, et al. (2007) Clinica 
Citation: Sarwar M, Persson JL (2011) The Protein Kinase A (PKA) Intracellular Pathway and Androgen Receptor: A Novel Mechanism Underlying the Castration-resistant and Metastatic Prostate Cancer. J Cancer Sci Ther S5:003. doi:10.4172/1948-5956.S5-003

implications of neuroendocrine differentiation in prostate cancer Prostate Cancer Prostatic Dis 10: 6-14

13. Merkle D, Hoffmann R (2011) Roles of cAMP and cAMP-dependent protein kinase in the progression of prostate cancer: cross-talk with the androgen receptor. Cell Signal 23: 507-515

14. Chin KV, Yang WL, Ravatn R, Kita T, Reitman E, et al. (2002) Reinventing the wheel of cyclic AMP: novel mechanisms of cAMP signaling. Ann NY Acad Sci 968: 49-64.

15. Taskén K, Skålhegg BS, Solberg R, Andersson KB, Taylor SS, et al. (1993) Novel isozymes of cAMP-dependent protein kinase exist in human cells due to formation of RI alpha-RI beta heterodimeric complexes. J Biol Chem 268 : 21276-21283.

16. Francis SH, Corbin JD (1999) Cyclic Nucleotide-Dependent Protein Kinases: Intracellular Receptors for cAMP and cGMP Action. Crit Rev Clin Lab Sci 36: 275-328.

17. Sands WA, Palmer TM (2008) Regulating gene transcription in response to cyclic AMP elevation. Cell Signal 20: 460-466.

18. Mayr B, Montminy M (2001) Transcriptional regulation by the phosphorylationdependent factor CREB. Nat Rev Mol Cell Biol 2: 599-609.

19. Sakamoto KM, Frank DA (2009) CREB in the pathophysiology of cancer: implications for targeting transcription factors for cancer therapy. Clin Cancer Res 15: 2583-2587.

20. Lugnier C (2006) Cyclic nucleotide phosphodiesterase (PDE) superfamily: A new target for the development of specific therapeutic agents. Pharmacol Ther 109: 366-398.

21. Richter W, Conti M (2004) The Oligomerization State Determines Regulatory Properties and Inhibitor Sensitivity of Type 4 cAMP-specific Phosphodiesterases. J Biol Chem 279: 30338-30348.

22. Abrahamsen H, Baillie G, Ngai J, Vang T, Nika K, et al. (2004) TCR- and CD28mediated recruitment of phosphodiesterase 4 to lipid rafts potentiates TCR signaling. J Immunol 173: 4847-4858.

23. Smith FD, Langeberg LK, Scott JD (2006) The where's and when's of kinase anchoring. Trends Biochem Sci 31 : 316-323.

24. Conti M, Richter W, Mehats C, Livera G, Park JY, et al. (2003) Cyclic AMP specific PDE4 Phosphodiesterases as Critical Components of Cyclic AMP Signaling. J Biol Chem 278: 5493-5496.

25. Neary CL, Nesterova M, Cho YS, Cheadle C, Becker KG, et al. (2004) Protein kinase $A$ isozyme switching: eliciting differential CAMP signaling and tumor reversion. Oncogene 23: 8847-8856.

26. Tortora G, Clair T, Katsaros D, Ally S, Colamonici O, et al. (1989) Induction of megakaryocytic differentiation and modulation of protein kinase gene expression by site-selective cAMP analogs in K-562 human leukemic cells. Proc Natl Acad Sci USA 86: 2849-2852.

27. Cho-Chung YS (1989) Site-Selective 8-Chloro- Cyclic Adenosine 3' $5^{\prime}$-Monophosphate as a Biologic Modulator of Cancer: Restoration of Normal Control Mechanisms. J Natl Cancer Inst 81: 982-987.

28. Cho-Chung YS (1990) Role of Cyclic AMP Receptor Proteins in Growth Differentiation, and Suppression of Malignancy: New Approaches to Therapy. Cancer Res 50: 7093-7100.

29. Cho-Chung YS, Nesterova MV (2005) Tumor reversion: protein kinase A isozyme switching. Ann NY Acad Sci 1058: 76-86.

30. Khor LY, Bae K, Al-Saleem T, Hammond EH, Grignon DJ, et al. (2008) Protein Kinase A RI- $\alpha$ Predicts for Prostate Cancer Outcome: Analysis of Radiation Therapy Oncology Group Trial 86-10. Int J Radiat Oncol Biol Phys 71: 13091315.

31. Richards JS, Rolfes Al (1980) Hormonal regulation of cyclic AMP binding to specific receptor proteins in rat ovarian follicles. Characterization by photoaffinity labeling. J Biol Chem 255: 5481-5489.

32. Cho YS, Kim MK, Tan L, Srivastava R, Agrawal S, et al. (2002) Protein Kinase A Rla Antisense Inhibition of PC3M Prostate Cancer Cell Growth: Bcl-
2 Hyperphosphorylation, Bax Up-Regulation, and Bad-Hypophosphorylation. Clin Cancer Res 8: 607-614.

33. Mani S, Goel S, Nesterova M, Martin RM, Grindel JM, et al. (2003) Clinica Studies in Patients with Solid Tumors using a Second-Generation Antisense Oligonucleotide (GEM®231) targeted against Protein Kinase A Type I. Ann NY Acad Sci 1002: 252-262.

34. Cho YS, Kim MK, Cheadle C, Neary C, Becker KG, et al. (2001) Antisense DNAs as multisite genomic modulators identified by DNA microarray. Proc Nat Acad Sci USA 98: 9819-9823.

35. Pollack A, Bae K, Khor LY, Al-Saleem T, Hammond ME, et al. (2009) The Importance of Protein Kinase A in Prostate Cancer: Relationship to Patien Outcome in Radiation Therapy Oncology Group Trial 92-02. Clin Cancer Res 15: $5478-5484$

36. Desiniotis A, Schafer G, Klocker H, Eder IE (2010) Enhanced antiproliferative and proapoptotic effects on prostate cancer cells by simultaneously inhibiting androgen receptor and cAMP-dependent protein kinase A. Int J Cancer 126 $775-789$

37. Goel S, Desai K, Macapinlac M, Wadler S, Goldberg G, et al. (2006) A phase I safety and dose escalation trial of docetaxel combined with GEM ${ }^{2} 231$, a second generation antisense oligonucleotide targeting protein kinase A R1a in patients with advanced solid cancers. Invest New Drugs 24: 125-134.

38. Agrawal S, Kandimalla ER, Yu D, Ball R, Lombardi G, et al. (2002) GEM 231, a second-generation antisense agent complementary to protein kinase A Rlalpha subunit, potentiates antitumor activity of irinotecan in human colon, pancreas prostate and lung cancer xenografts. Int J Oncol 21: 65-72

39. Goto T, Matsushima H, Kasuya Y, Hosaka Y, Kitamura T, et al. (1999) The effect of papaverine on morphologic differentiation, proliferation and invasive potential of human prostatic cancer LNCaP cells. Int J Urol 6: 314-319.

40. Kasbohm EA, Guo R, Yowell CW, Bagchi G, Kelly P, et al. (2005) Androgen receptor activation by $\mathrm{G}(\mathrm{s})$ signaling in prostate cancer cells. J Biol Chem 280 11583-11589.

41. Kashiwagi E, Shiota M, Yokomizo A, Itsumi M, Inokuchi J, et al. (2011) Downregulation of phosphodiesterase 4B (PDE4B) activates protein kinase A and contributes to the progression of prostate cancer. The Prostate [Early View (Online Version of Record published before inclusion in an issue)].

42. Scanlan MJ, Gure AO, Jungbluth AA, Old LJ, Chen YT (2002) Cancer/testis antigens: an expanding family of targets for cancer immunotherapy. Immuno Rev 188: 22-32.

43. Chiriva-Internati M, Yu Y, Mirandola L, D'Cunha N, Hardwicke F, et al. (2011) Identification of AKAP-4 as a new cancer/testis antigen for detection and immunotherapy of prostate cancer. Prostate $\square$ 72: 12-23.

44. Xiao X, Li BX, Mitton B, Ikeda A, Sakamoto KM (2010) Targeting CREB for cancer therapy: friend or foe. Curr Cancer Drug Targets 10: 384-391.

45. Wu D, Zhau HE, Huang WC, Iqbal S, Habib FK, et al. (2007) cAMP-responsive element-binding protein regulates vascular endothelial growth factor expression: implication in human prostate cancer bone metastasis. Oncogene 26: $5070-5077$

46. Chung S, Furihata M, Tamura K, Uemura M, Daigo Y, et al. (2009) Overexpressing $\mathrm{PKIB}$ in prostate cancer promotes its aggressiveness by linking between PKA and Akt pathways. Oncogene 28: 2849-2859.

47. Kasbohm EA, Guo R, Yowell CW, Bagchi G, Kelly P, et al. (2005) Androgen Receptor Activation by G(s) Signaling in Prostate Cancer Cells. J Biol Chem 280: 11583-11589

48. Wang X, Lu XA, Song X, Zhuo W, Jia L, et al. (2011) Thr90 phosphorylation of Hsp90alpha by Protein Kinase A Regulates Its Chaperone Machinery. Biochem J 441: 387-397.

49. Nazareth LV, Weigel NL (1996) Activation of the Human Androgen Recepto through a Protein Kinase A Signaling Pathway. J Biol Chem 271: 19900-19907.

50. Sadar MD (2009) The Role of Cyclic AMP in Regulating the Androgen Receptor Androgen Action in Prostate Cancer. In: Mohler J, Tindall D (Eds) Springer New York 465-503. 
Citation: Sarwar M, Persson JL (2011) The Protein Kinase A (PKA) Intracellular Pathway and Androgen Receptor: A Novel Mechanism Underlying the Castration-resistant and Metastatic Prostate Cancer. J Cancer Sci Ther S5:003. doi:10.4172/1948-5956.S5-003

51. Kim J, Jia L, Stallcup MR, Coetzee GA (2005) The role of protein kinase A pathway and cAMP responsive element-binding protein in androgen receptormediated transcription at the prostate-specific antigen locus. J Mol Endocrinol 34: 107-118.

52. Mizokami A, Yeh SY, Chang C (1994) Identification of 3',5'-cyclic adenosine monophosphate response element and other cis-acting elements in the human androgen receptor gene promoter. Mol Endocrinol 8: 77-88.

53. Majumder PK, Sellers WR (2005) Akt-regulated pathways in prostate cancer Oncogene 24: 7465-7474.

54. Hennessy BT, Smith DL, Ram PT, Lu Y, Mills GB (2005) Exploiting the PI3K/ AKT Pathway for Cancer Drug Discovery. Nat Rev Drug Discov 4: 988-1004

55. De Gregorio G, Coppa A, Cosentino C, Ucci S, Messina S, et al. (2006) The p85 regulatory subunit of PI3K mediates TSH-cAMP-PKA growth and survival signals. Oncogene 26: 2039-2047.

56. Fang X, Yu SX, Lu Y, Bast RC, Woodgett JR, et al. (2000) Phosphorylation and inactivation of glycogen synthase kinase 3 by protein kinase A. Proc Natl Acad Sci USA 97: 11960-11965.

57. Vashchenko N, Abrahamsson PA (2005) Neuroendocrine Differentiation in Prostate Cancer: Implications for New Treatment Modalities. Eur Urol 47: 147155

58. Abrahamsson PA (1999) Neuroendocrine cells in tumour growth of the prostate Endocr Relat Cancer 6: 503-519.

59. Yuan TC, Veeramani S, Lin MF (2007) Neuroendocrine-like prostate cancer cells: neuroendocrine transdifferentiation of prostate adenocarcinoma cells. Endocr Relat Cancer 14: 531-547.

60. Sun Y, Niu J, Huang J (2009) Neuroendocrine differentiation in prostate cancer. Am J Transl Res 1: 148-162.

61. di Sant'Agnese PA (2001) Neuroendocrine differentiation in prostatic carcinoma: an update on recent developments. Ann Oncol 12: S135-S140.

62. Cox ME, Deeble PD, Bissonette EA, Parsons SJ (2000) Activated 3',5'-Cyclic AMP-dependent Protein Kinase Is Sufficient to Induce Neuroendocrine-like
Differentiation of the LNCaP Prostate Tumor Cell Line. J Biol Chem 275 $13812-13818$.

63. Deeble PD, Cox ME, Frierson HF, Sikes RA, Palmer JB, et al. (2007) Androgen-Independent Growth and Tumorigenesis of Prostate Cancer Cells Are Enhanced by the Presence of PKA-Differentiated Neuroendocrine Cells. Cancer Res 67: 3663-3672.

64. Khor LY, Bae K, Al-Saleem T, Hammond EH, Grignon DJ, et al. (2008) Protein Kinase A RI- $\alpha$ Predicts for Prostate Cancer Outcome: Analysis of Radiation Therapy Oncology Group Trial 86-10. Int J Radiat Oncol Biol Phys 71: 13091315

65. Pollack A, Bae K, Khor LY, Al-Saleem T, Hammond ME, et al. (2009) The importance of protein kinase A in prostate cancer: Relationship to patien outcome in Radiation Therapy Oncology Group trial 92-02. Clinical Cancer Res 15: $5478-5484$

66. Rosser C, Gaar M, Porvasnik S (2009) Molecular fingerprinting of radiation resistant tumors: Can we apprehend and rehabilitate the suspects? BMC Cancer $9: 225$.

67. Bonkhoff H (2012) Factors Implicated in Radiation Therapy Failure and Radiosensitization of Prostate Cancer. Prostate Cancer 2012: 1-12.

68. Hensley HH, Hannoun-Levi JM, Hachem P, Mu Z, Stoyanova R, et al. (2011) PKA knockdown enhances cell killing in response to radiation and androgen deprivation. Int J Cancer 128: 962-973.

69. Katsaros D, Tortora G, Tagliaferri P, Clair T, Ally S, et al. (1987) Site-selective cyclic AMP analogs provide a new approach in the control of cancer cell growth. FEBS Lett 223: 97-103.

70. Lucchi S, Calebiro D, de Filippis T, Grassi ES, Borghi MO, et al. (2011) 8-Chloro-Cyclic AMP and Protein Kinase A I-Selective Cyclic AMP Analogs Inhibit Cancer Cell Growth through Different Mechanisms. PLoS ONE 6 e20785

71. Shankar DB, Cheng JC, Kinjo K, Federman N, Moore TB, et al. (2005) The role of CREB as a proto-oncogene in hematopoiesis and in acute myeloid leukemia. Cancer Cell 7: 351-362.
This article was originally published in a special issue, Cancer Research: Clinical \& Experimental handled by Editor(s). Dr. Richard D. Finkelman, AstraZeneca LP Clinical, USA; Dr. Jimmy Thomas Efird, University of North Carolina, USA; Dr. Yanming Wang, Case Western Reserve University, USA 